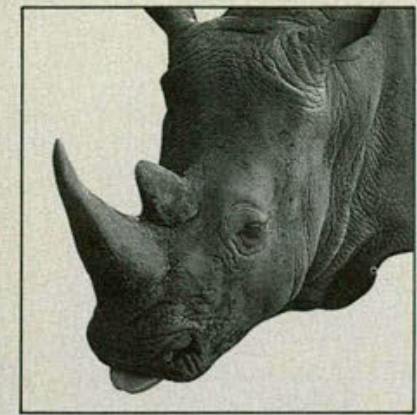

It's on its way to your office.

BROMFED Timed-release Capsules (brompheniramine maleate $12 \mathrm{mg}$ and pseudoephedrine $\mathrm{HCl} 120 \mathrm{mg}$ )

BROMFED-PD ${ }^{\circledR}$ Timed-release Capsules (brompheniramine maleate $6 \mathrm{mg}$ and pseudoephedrine $\mathrm{HCl} 60 \mathrm{mg}$ )

Brief Summary

CONTRAINDICATIONS Hypersensitivity to any of the in gredients. Also contraindicated in patients with severe hypertension, severe coronary artery disease, patients on coma, urinary retention, peptic ulcer and during an asthmatic attack.

WARNINGS Considerable caution should be exercised WARNINGS Considerable caution should be exercised in patients with hypertension, diabetes mellitus, ischemic
heart disease, hyperthyroidism, increased intraocular pressure and prostatic hypertrophy. The elderly ( 60 years or older) are more likely to exhibit adverse reactions.

Antihistamines may cause excitability, especially in children. At dosages higher than the recommended dose, nervousness, dizziness or sleeplessness may occur.

PRECAUTIONS General: Caution should be exercised in patients with high blood pressure, heart disease, dia in patients with high blood pressure, heart disease, diabetes or thyroid disease. The antihistamine in this produc may exhibit additive
including alcohol.

Information for Patients: Antihistamines may cause drowsiness and ambulatory patients who operate machinery or motor vehicles should be cautioned accordingly.

Drug Interactions: MAO inhibitors and beta adrenergic blockers increase the effects of sympathomimetics. Sym pathomimetics may reduce the antihypertensive effects of methyldopa, mecamylamine, reserpine and veratrum alkaoids. Concomitant use of antihistamines with alcohol and other CNS depressants may have an additive effect.

Pregnancy: The safety of use of this product in pregnancy has not been established.

ADVERSE REACTIONS Adverse reactions include drowsiness, lassitude, nausea, giddiness, dryness of the mouth, blurred vision, cardiac palpitations, flushing, in creased irritability or excitement (especially in children).

Dosage and Administration

BROMFED* CAPSULES Adults and children over 12 years of age: 1 capsule every 12 hours.

BROMFED-PD* CAPSULES Children 6 to 12 years of age: 1 capsule every 12 hours. Adults and children over 12 years of age: 1 or 2 capsules every 12 hours.

BROMFED ${ }^{3}$ TABLETS Adults and children 12 and over: One tablet every 4 hours not to exceed 6 doses in 24 hours. Children 6 to 12 years: One-half tablet every 4 hours not to exceed 6 doses in 24 hours. Do not give to children under 6 years except under the advice and supervision of a physician

CAUTION: FEDERAL (U.S.A) LAW PROHIBITS DISPENSING WITHOUT A PRESCRIPTION.

\section{Distributed by}

\section{Muro}

Pharmaceutical, Inc.

Tewksbury, MA 01876-9987

letters

\section{'Loopholes' weaken hospital accreditation policy}

\section{To the Editor:}

I read your editorial "Hospital accreditation pounds another 'nail' in the 'smoking' coffin $(J A O A 1991 ; 91: 1171)$ with pleasure. However, this pleasure soon turned to dismay as I realized that the tough new accreditation policy of the American Osteopathic Association is full of holes for the convenience of nearly anyone who may not want to contribute to the establishment of a smokeless society.

Specifically, the policy states that hospitals may make exceptions to their policy where "abrupt cessation of smoking would be detrimental to the patient." I tried to think of those medical conditions that would be exacerbated by abrupt cessation of cigarette smoking and thereby cause harm to the patient. My list was very short.

Another provision in this policy states that hospitals "will segregate, where practi- cal, patients authorized to smoke from nonsmoking patients." When is it not practical to segregate smokers from nonsmokers in any environment, especially that of a hospital? Even the US military, traditionally a hard-drinking, heavy-smoking group, now has established totally smokefree hospitals. Anyone in a military hospital must now step outside in designated areas to smoke. Why can't the rest of the hospitals make this logical transition? In my opinion, the potential for a malpractice suit lies behind this failure to segregate.

In short, what began and ended as a triumphant proclamation, seemed, in the middle, to be little more than loopholed lip service. I hope that those persons in politically powerful positions can, in the future, adopt a policy more consistent with those of us dedicated to saving and improving lives.

LT JAMES C. HORSPOOL, DO

Department of Pediatrics

Walter Reed Army Medical

Center

Washington, DC 


\section{For hypertensives over 55}

\section{The golden age of $A C E$ therapy}

ONCE DAILY

for many patients

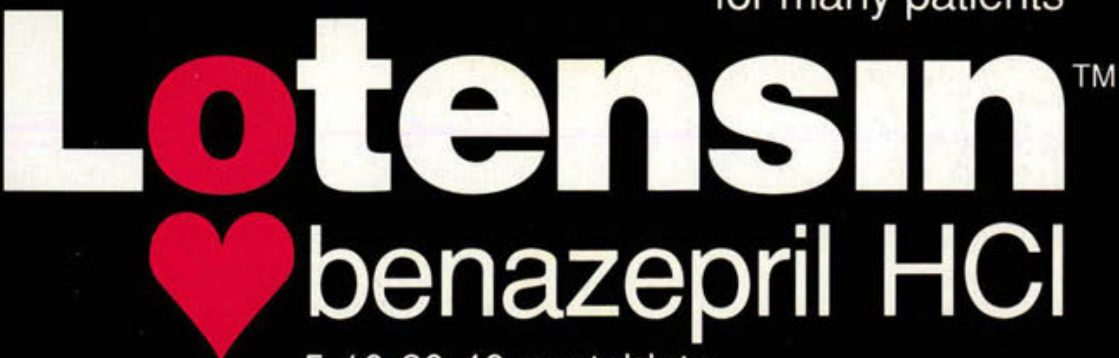

$5 \cdot 10 \cdot 20 \cdot 40-\mathrm{mg}$ tablets 


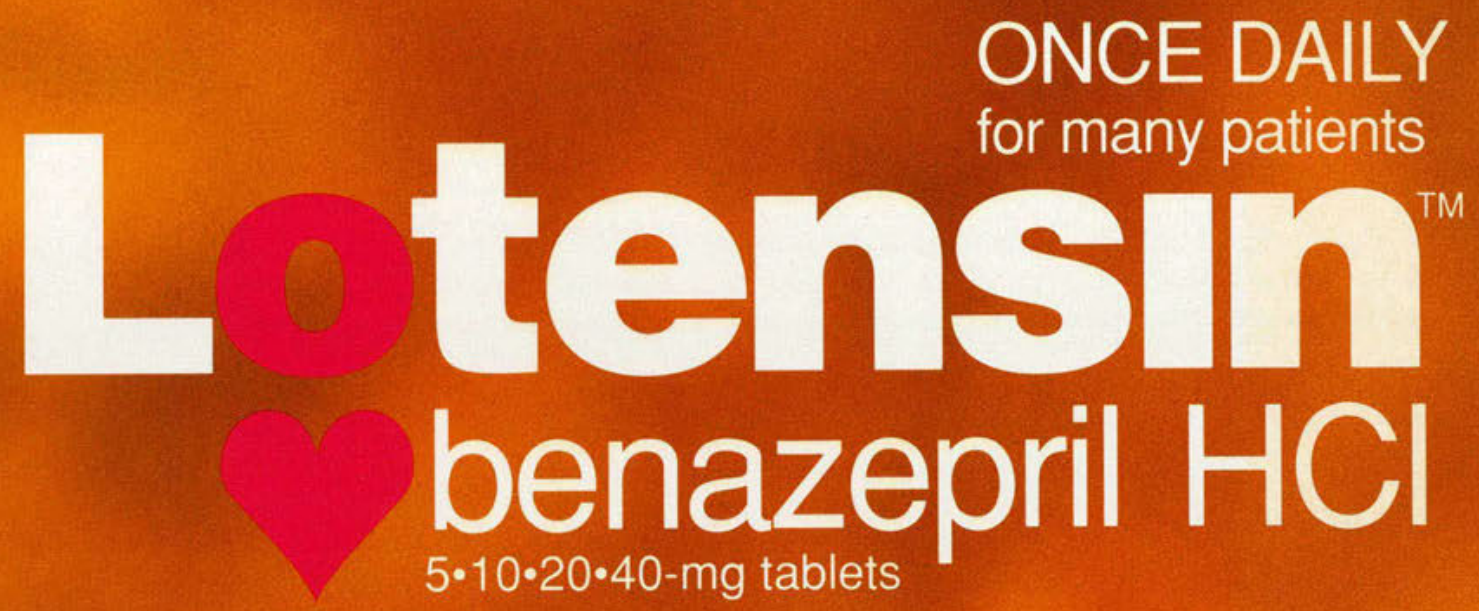

\section{ACE inhibition without age inhibitio}

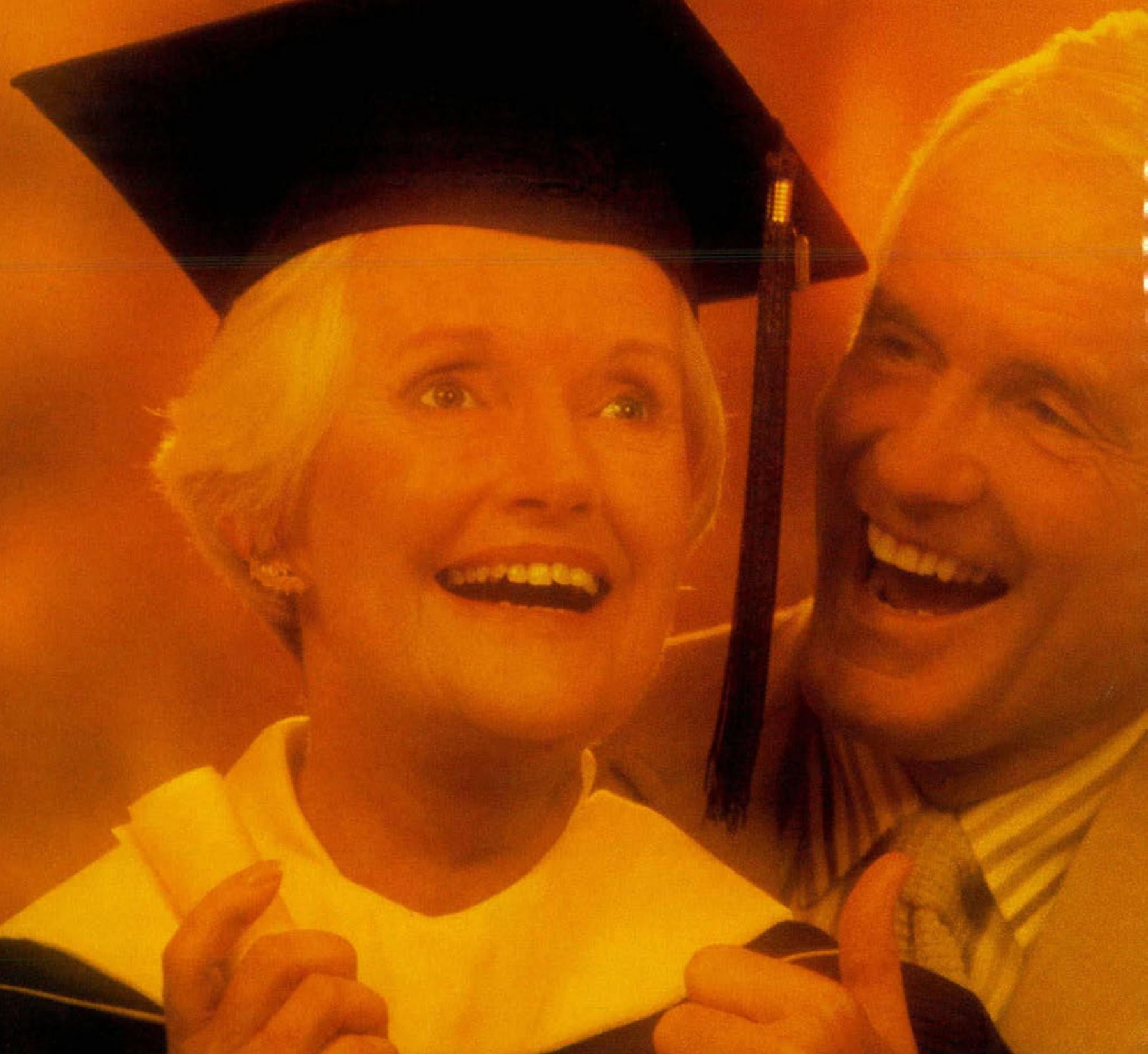




\section{In hypertension}

\section{Once-Daily Efficacy \\ Plus}

\section{Important Safety Benefits ...}

\section{Especially in Patients Over 55}

Safe and well-tolerated regardless of age. ${ }^{2}$ In controlled clinical trials, overall incidence of adverse events comparable to placebo. Incidence of cough statistically significant. ${ }^{3}$

Same starting dose even in patients with mild-to-moderate renal impairment $\left(\mathrm{Cl}_{\mathrm{cr}} \geq 30 \mathrm{~mL} / \mathrm{min}\right){ }^{4}$

No important pharmacokinetic interactions observed when coadministered with:

- digoxin ${ }^{3}$

- propranolol $^{3}$

- furosemide ${ }^{6}$
- warfarin 5

- cimetidine $^{3}$

- chlorthalidone ${ }^{3}$
- naproxen ${ }^{3}$

- atenolol ${ }^{3}$

- hydrochlorothiazide ${ }^{7}$

The evaluation of any hypertensive patient should include renal function studies. ACE inhibition may cause changes in renal function in susceptible individuals. Lotensin is contraindicated in patients who are hypersensitive to this product or to any other ACE inhibitor. Angioedema has been reported in patients receiving ACE inhibitors. Please consult brief summary of Prescribing Information, including the "Drug Interactions and Precautions - Impaired Renal Function" sections on last page.

C I B A 


\section{ACE inhibition without age inhibition}

\section{Lotensin ${ }^{\circledR}$}

benazepril hydrochloride

Tablets

\section{BRIEF SUMMARY (FOR FULL PRESCRIBING}

INFORMATION, SEE PACKAGE INSERT

dicated for the treatment of

hypertension. It may be used alone or in combination with thiazide

In using Lotensin, consideration should be given to the fact that another angiotensin-converting enzyme inhibitor, captopril, has caused agranulocytosis, particularly in patients with renal impairment or Lotensin does not have a similar risk (see WARNINGS).

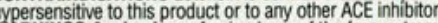

WARNINGS-Angioedema Angioedema of the face, extremities, lips tongue, glottis, and larynx has been reported in patients treated with angiotensin-converting enzyme inhibitors. In U.S. clinical trials,
symptoms consistent with angioedema were seen in none of the symptoms consistent with angioedema were seen in none of the
subjects who received placebo and in about $0.5 \%$ of the subjects who subjects who received placebo and in about $0.5 \%$ of the subjects who be fatal. If laryngeal stridor or angioedema of the face, tongue, or be tatal. If laryngeal stridor or angioedema of the lace, tongue, or glottis occurs, treatment with Lotensin should be discontin involvement of the tongue, glottis, or larynx, likely to cause airway injection $1: 1000(0.3 \mathrm{~mL}$ to $0.5 \mathrm{~mL})$ should be promptty

administered (see ADVERSE REACTIONS)

Hypotension Lotensin can cause symptomatic hypotension. Like other ACE inhibitors, benazepril has been only rarely associated with hypotension in uncomplicated hypertensive patients. Symptomatic and/or salt-depleted as a result of prolonged diuretic therapy, dietary salt restriction, dialysis, diarrhea, or vomiting. Volume-and/or saltIn patients with congestive heart falure, with or without associated In patients with congestive heart failure, with or without associlive
nal insufficiency. ACE inhibitor therapy may cause excessive hypotension, which may be associated with oliguria or azotemia and. rarely, with acute renal falure and death. In such patients, Lotensin therapy should be started under close medical supervision; they should dose of benazepril or diuretic is increased.

If hypotension occurs, the patient should be placed in a supine position, and, if necessary, treated with intravenous infusion of physiological saline. Lotensin treatment usually can be continued fllowing restoration of blood pressure and volume. Neutropenia/Agranulocylosis Another angiotensin-converting enzyme marrow depression, rarely in uncomplicated patients, but more frequently in patients with renal impairment, especially if they also hav a collagen-vascular disease such as systemic lupus erythematosus or scleroderma. Available data from clinical trials of benazepril are insufficient to show that benazepril does not cause agranulocytosis at similar rates. Monitoring of white blood cell counts should be

considered in patients with collagen-vascular disease, especially if the disease is associated with impaired renal function

Fetal/Neonatal Morbidity and Mortality ACE inhibitors can cause feta and neonatal morbidity and mortality when administered to pregnant

When ACE inhibitors have been used during the second and third trimesters of pregnancy, there have been reports of neonata

hypotension, renaa falure, skull hypoplasia, and death. Oligohydramnios has also been reported, presumably resulting from decreased te contractures, craniofacial malformations, hypoplastic lung contractures, craniofacial malformations, hypoplastic lung patent ductus arteriosus have been reported, although it is not clear whether these occurrences were due to the ACE-inhibitor exposure or to the mother's underlying disease.

It is not known whether exposure limited to the first trimester can

adversely affect fetal outcome.
A patient who becomes pregnant while taking ACE inhibitors, or Who takes ACE inhibitors when already pregnant, should be apprised the potential hazard to her fetus. If she continues to receive ACE digohydramnios. When oligohydramnios is found, ACE inhibitors should generally be discontinued.

Infants with histories of in utero exposure to ACE inhibitors should
. be closely observed for hypotension, oliguria and hypokalemia if oliguria occurs, attention should be directed toward support of blood pressure and renal perfusion. Benazepril could theoretically be removed from the neonatal circulation by exchange transfusion, but $n$

experience with this procedure has been reported.
At doses that did not induce maternal toxicity, no embryotoxic fetotoxic or teratogenic effects of Lotensin were seen in studies of pregnant rats, mice, and rabbits. On a $\mathrm{mg} / \mathrm{m}^{2}$ basis, the doses used in
these studies were 60 times (in rats), 9 times (in mice), and more than 0.8 times (in rabbits) the maximum recommended human dose assuming a $50 \mathrm{~kg}$ woman). On a mg/kg basis these multiples are 300 the maximum recommended human dose. the renin-angioensin-aldosterone system chanoes in renal function may be anticipated in susceptible individuals. In patien with severe congestive heart failure whose renal function may depend
on the activity of the renin-angiotensin-aldosterone system, treatment with angiotensin-converting enzyme inhibitors, including Lotensin, may be associated with oliguria and/or progressive azotemia and
(rarely) with acute renal failure and/or death. In a small study of hypertensive patients with renal artery stenosis in a solitary kidney or bilateral renal artery stenosis, treatment with Lotensin was associated with increases in blood urea nitrogen and serum creatinine; these increases were reversible upon discontinuation of Lotensin or diuretic therapy, or both. When such patients are treated with ACE inhibito
renal function should be monitored during the first few weeks of therapy. Some hypertensive patients with no apparent preexisting ren vascular disease have developed increases in blood urea nitrogen and serum creatinine, usually minor and transient, especially when Lotensin has been given concomitantly with a diuretic. This is more likely to occur in patients with preexisting renal impairment. Dosage required. Evaluation of the hypertensive patient should always include assessment of renal function (see DOSAGE AND ADMINISTRATION). Hyperkalemia: In clinical trials, hyperkalemia (serum potassium at least $0.5 \mathrm{mE}$ E $\mathrm{L}$ greater than the upper limit normal) occurred in approximately $1 \%$ of hypertensive patients receiving Lotensin. In most cases, these were isolated values which resolved despite continued therapy. Risk factors for the development
of hyperkalemia include renal insurficiency, diabetes mellitus, and the
concomitant use of potassium-sparing diuretics, potassium, supplements, and/or potassium-containing salt substitutes, which supplements, and/or potassium-containing salt substitutes,
should be used cautiously, If at all, with Lotensin (see Drug Interactions). Impaired Liver Function: In patients with hepatic dysfunction due to cirrhosis, levels of benazeprilat are essentially during anesthesia with agents that produce hyportension, benazepr will block the angiotensin II formation that could otherwise occur secondary to compensatory renin release. Hypotension that occurs a a result of this mechanism can be corrected by volume expansion Information for Patients - Angioedema: Angioedema, including
laryngeal edema, can occur with treatment with ACE inhibitors, laryngeal edema, can occur with treatment with ACE inhibitors,
especially following the first dose. Patients should be so advised and told to report immediately any signs or symptoms suggesting
angioedema (swelling of tace, eyes, lips, or tongue, or difficulty in breathing) and to take no more drug until they have consulted with the prescribing physician. Symptomatic Hypotension: Patients should be cautioned that lightheadedness can occur, especially during the first days of therapy, and it should be reported to the prescribing physicia Patients should be told that if syncope occurs, Lotensin should be discontinued until the prescribing physician has been consulted. excessive perspiration, diarrhea, or vomiting can lead to an excessive fall in blood pressure, with the same consequences of lightheadedness
and possible syncope. Hyperkalemia: Patients should be told not to use potassium supplements or salt substitutes containing potassium whoult consulting the prescribing physician. Neutropenia: Patients throat fever) which could be a sign of neutropenia

Drug Interactions - Diuretics: Patients on diuretics, especially those in whom diuretic therapy was recently instituted, may occasionally experience an excessive reduction of blood pressure after initiation therapy with Lotensin. The possibility of hypotensive effects with increasing the salt intake prior to initiation of treatment with Lotensin. If this is not possible, the starting dose should be reduced (see
DOSAGE AND ADMINISTRATION). Potassium Supplements and DOSAGE AND ADMINISTRATION). Potassium Supplements and
Potassium-Sparing Diuretic: Lotensin can attenuate potassium loss caused by thiazide diuretics. Potassium-sparing diuretics (spironolactone, amiloride, triamterene, and others) or potassium concomitant use of such agents is indicated, they should be given with caution, and the patient's serum potassium should be monitored frequently. Oral Anticoagulants: Interaction studies with warfarin and acenocoumarol falied to identify any clinically important effects on the serum concentrations or clinical effects of these anticoagulants. Lithium: Increased serum lithium levels and symptoms of lithium
toxicity have been reported in patients receiving ACE inhibitors during therapy with lithium. These drugs should be coadministered with caution, and frequent monitoring of serum lithium levels is recommended. If a diuretic is also used, the risk of lithium toxicity may be increased. Other: No clinically important pharmacokinetic
interactions occurred when Lotensin was administered concomitantly interactions occurred when Lotensin was administered concom
with hydrochlorothiazide, chlorthalidone, furosemide, digoxin, propranolol, atenolol, naproxen, or cimetidine propranolol, atenolol, naproxen, or cimetidine.
Lotensin has been used concomitantly with beta-adrenergic blocking agents, calcium-channel-blocking agents, diuretics, digoxin and hydralazine, without evidence of clinically important adverse interactions. Benazeprii, like other ACE inhibitors, has had less than additive effects with beta-adrenergic blockers, presumably because

angiotensin system. of carcinogenicity was found when benazepril was administered by
gavage to rats and mice for up to two years at doses of up to $150 \mathrm{~m}$ gavage to rats and mice for up to two years at doses of up to $150 \mathrm{mg}$
$\mathrm{kg} / \mathrm{day}$. When compared on the basis of body weights, this dose is 110 times the maximum recommended human dose. When compared on the basis of body surtace areas, this dose is 18 and 9 times
mice, respectively) the maximum recommended human dose (calculations assume a patient weight of $60 \mathrm{~kg}$ ). No mutagenic activity activation), in an in vitro test in bacteria (With or without metabo mammalian cells, or in a nucleus anomaly test. In doses of $50-500$ $\mathrm{mg} / \mathrm{kg} / \mathrm{day}(6-60$ times the maximum recommended human dose based on $\mathrm{mg} / \mathrm{m}^{2}$ comparison and $37-375$ times the maximum
recommended human dose based on a mg/kg comparison), Lotensin had no adverse effect on the reproductive performance of male and Pregnancy Category D See WARNINGS, FetalNeonatal Morbidity and Nursing Mothers Minimal amounts of unchanged benazepril and of benazeprilat are excreted into the breast milk of lactating women would receive less than $0.1 \%$ of the $\mathrm{mg} / \mathrm{kg}$ maternal dose of Geriatric Use Of the total number of patients who received benazepri in U.S. clinical studies of Lotensin, $18 \%$ were 65 or older while $2 \%$ were 75 or older. No overall differences in effectiveness or safety were observed between these patients and younger patients, and other reported clinical expenience has not identified differences in respons some older individuals cannot be ruled out Pediatric Use Satety and effectiveness in children have not been ADVERSE REACTIONS Lotensin has been evaluated for safety in over

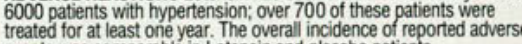
events was comparable in Lotensin and placebo patients.

The reported side effects were generally mild and transient, and there was no relation between side effects and age, duration of there was or total dosage within the range of 2 to $80 \mathrm{mg}$

approximately $5 \%$ of U.S. patients treated with Lotensin and in $3 \%$ of patients treated with placebo.

The most common reasons for discontinuation were headache $0.6 \%)$ and cough $(0.5 \%$

In placebo-controlled trials, cough was reported by 68 of 2004 benazepril-treated patients $(3.4 \%)$ and by 7 of 525 placebo-treated st $1 \%$ that was significantly more frequent in benazepril-treated th placebo.

drug that occurred in U.S. controlled trials in mobably related to study treated with Lotensin are shown belOW.
PATIENTS IN U.S. PLACEBO-CONTROLLED STUDIES

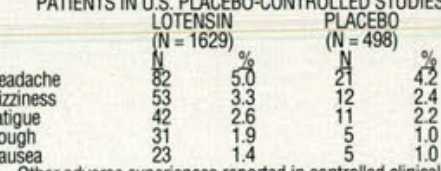

Other adverse experiences reported in controlled clinical trials (in less than $1 \%$ of benazepril patients), and rarer events seen in postmarketing experience, include the following (in some, a causal hypotension to drug use is uncertain): Cardiovascular: Symptomatic $0.4 \%$, and syncope in $0.1 \%$; these reactions led to discontinuation of therapy in 4 patients who had received benazepril monotherapy and in PRECAUTIONS and WARNINGS). Other reports included angina pectoris, palpitations, and peripheral edema. Renal: Of hypertensive patients with no apparent preexisting renal disease, abouf $2 \%$ have sustained increases in serum creatinine to at least $150 \%$ of their
baseline values while receiving Lotensin, but most of these increases have disappeared despite continuing treatment. A much smaller traction of these patients (less thal irea nitrogen and serum (usually transient) increases in blood urea nitrogen and serum creatinine. Angioedema: Angioedema has been reported in patients with benazepril, $0.5 \%$ of patients experienced edema of the lips or face without other manifestations of angioedema. Angioedema associated with laryngeal edema and/or shock may be fatal. If angioedema
face, extremities, lips, tongue, or glottis and/or larynx occurs. treatment with Lotensin should be discontinued and appropriate therapy instituted immediately (see WARNINGS). Gastrointestinal: Constipation, gastritis, vomiting, and melena. Dermatologic: Appare
hypersensitivity reactions (manifested by dermatitis, prurtis, or rash) and flushing. Neurologic and Psychiatric: Anxiety, decreased libido, hypertonia, insomnia, nervousness, and paresthesia. Other: Arthral gi
arthritis, asthenia, asthma, bronchitis, dyspnea, impotence, infection, myalgia, sinusitis, sweating, and urinary tract infection. Clinical Laboratory Test Findings

Creatinine and Blood Urea Nitrogen: Of hypertensive patients with no apparent preexisting renal disease, about $2 \%$ have sustained increase receiving creatinine to at least $150 \%$ of their baseline values while despite continuing treatment. A much smaller fraction of these patien (less than $0.1 \%$ ) developed simultaneous (usually transient) increases in blood urea nitrogen and serum creatinine. None of these increases required discontinuation of treatment. Increases in these laboratory
values are more likely to occur in patients with renal insulficiency or those pretreated with a diuretic and, based on experience with other ACE inhibitors, would be expected to be especially likely in patients with renal artery stenosis (see PRECAUTTIONS, General). Potassium: Since benazepril decreases aldosterone secretion, potassium-sparing diuretics should be given with caution, and the patient's serum potassium should be monitored frequently (see and a decrease of $5 \mathrm{~g} / \mathrm{dL}$ ) were rare, occurring in only 1 of 2014 patients receiving Lotensin alone and in 1 of 1357 patients receiving Lotensin plus a diuretic. No U.S. patients discontinued treatment
because of decreases in hemogiobin. Other (causal relationships 
ONCE DAILY

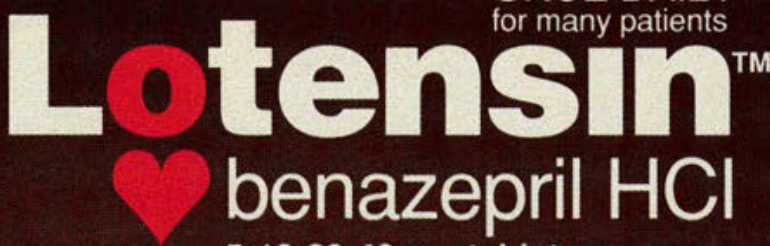

$5 \cdot 10 \cdot 20 \cdot 40-\mathrm{mg}$ tablets

CIBA Pharmaceutical Company has taken the high cost of quality health care to heart by pricing Lotensin 10-44\% below Capoten ${ }^{\circ}$,

Vasotec ${ }^{\star}$, Zestril ${ }^{\oplus}$,

unknown): Clinically important changes in standard laboratory tests enzymes, serum bilinubin uric acid and blood glucose have been reported, as have scattered incidents of hyponatremia, electrocardio graphic changes, leukopenia, eosinophila, and proteinuria. In U.S. trials, less than $0.5 \%$ of patients discontinued treatment because of laboratc OVERDOSAGE Single oral doses of $3 \mathrm{~g} / \mathrm{kg}$ benazepril were associated with significant lethality in mice. Rats however, tolerated single oral
doses of up to $6 \mathrm{~g} / \mathrm{kg}$. Reduced activity was seen at $1 \mathrm{~g} / \mathrm{kg}$ in mice and at
$5 \mathrm{~g} / \mathrm{kg}$ in rats. Human overdoses of benazepril have not been reported, but common manifestation of human benazepril overdosage is likely to be hypotension

Laboratory determinations of serum levels of benazepril and its metabolites are not widely available, and such determinations hav any event, no established role in the management of benazepri
overdose. No data are available to suggest physiological maneuvers (e.g. maneuvers to change the $\mathrm{pH}$ of the urine) that might accelerate
elimination of benazepril and its metabolites. Benazeprilat can be removed from the body by dialysis, but this intervention should rarely, if ever, be required.

Angiotensin II could presumably serve as a specific antagonistessentially unavailable outside of scattered research facilities the hypotensive effect of benazepril is achieved through vasod Because effective hypovolemia it is reasonable to treat benazepril overdose by infusion of normal saline solution. patients not receiving a diuretic is $10 \mathrm{mg}$ once-a-day. The usual maintenance dosage range is $20-40 \mathrm{mg}$ per day administered as a single dose or in two equally divided doses. A dose of $80 \mathrm{mg}$ gives an increased response, but experience with this dose is limited. The divide regimen was more effective in controlling trough (pre-dosing) blooc adjustment should be based on measurement of peak (2-6 hours after dosing) and trough responses. If a once-daily regimen does not give adequate trough response an increase in dosage or divided with Lotensin alone, a diuretic can be adde

Total daily doses above $80 \mathrm{mg}$ have not been evaluated Concomitant administration of Lotensin with potassiun supplements, potassium salt substitutes, or potassiumsparing diuretics can lead to increases of serum potassiun

(See PRECAUTIONS). symptomatic hypotension occasionally can occur following the initial dose of Lotensin. To reduce the likelihood of hypotension, the
diuretic should, if possible, be discontinued two to three days prior to
beginning therapy with Lotensin (see WARNINGS). Then, if blood pressure is not controlled with Lotensin alone, diuretic therapy should be If the diuretic cannot be discontinued, an initial dose of $5 \mathrm{mg}$ Lotensin should be used to avoid excessive hypotension Dosage Adjustment in Renal Impairment for patients with a creatinine clearance $<30 \mathrm{~mL} / \mathrm{min} / 1.73 \mathrm{~m}^{2}$ (serum
creatinine $>3 \mathrm{mg} / \mathrm{dL}$ ), the recommended initial dose is $5 \mathrm{mg}$ Lotensin once daily. Dosage may be titrated upward until blood pressure is

\section{B A}

Dist. by:

CIBA Pharmaceutical Company

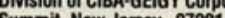

mmit, New Jersey 07901

terences:

Whalen J, Skalky C, deSilva J, Weber M. Peak and trough effects of 3 once daily dose levels of benazepril in mild-moderate hypertension. Am J Hypertens. 1989:2(part 2):45A. Gomez $\mathrm{HJ}$, Giazer R, Mallows S, deSilva J.B Benazepril in the treatment of older and elderly hypertensive patients. Royd Society or Medicine Services International Congress and . Data on file, ClBA Pharmaceutical Company.
Kaiser G. Ackermann R, Sioufi A. Pharmacokinetics of a new angiotensin-converting enzyme inhibitor, benazepril hydrochloride. in special populations. Am Heart J. 1989:117: 746 . Van Hecken A. De Lepeleire I, Verbesselt R R et al Efftect of benazepril, a converting enzyyme inhibitor, on plasma levels an activity of acenocoummarol and warlarin. Int $J$ Clin Pharmacol
and Res. 1989: 8 -315-319.

De Lepeleire I, Van Hecken A, Verbesselt R et al. Interaction between furosemide and the converting enzot al. Interaction inhibitor benazepril in healthy volunteers. Eur $J$ Clin Pharmacol. benazepril in health

Kaiser G. Dresse A. Ackermann R et al. Interaction between benazepril hydrochlorothiazide and hydrochloroth hiaziade in healthy volunteers. Eur Heart J. 1989:10(suppl): 118

... by promising a

lifetime of savings with and Prinivil ${ }^{\text {** }}$ and...

\section{The Lotensin}

\section{Lifetime Guarantee}

- Lotensin Lifetime Guarantee Price

- All Lotensin Dosages Priced the Same

Ask your CIBAVSummit Sales Representative for details about Lotensin and the Lotensin Lifetime Guarantee.

'Based on Red Book (10/91) Average Wholesale Price (AWP). Price comparisons reflect the most common dosing schedules for each product, not simply a tablet to tablet comparison.

TCIBA Pharmaceutical Company has established the Lotensin Lifetime Guarantee for patients who are started on Lotensin and enrolled in the program between 7/19/91 and 7/18/92.

As part of the Lotensin Lifetime Guarantee, the Lotensin Lifetime Guarantee Price was based on the Average Wholesale Price at the time of product introduction plus a standard pharmacy markup. This price is guaranteed for as long as the patient is on Lotensin therapy. Patients who enroll will be rebated the difference between the Lifetime Guarantee Price and any higher price that can be documented as paid with an original receipt. Because retail prices may vary, a maximum rebate has been established. For details, call 1-800-621-0021.

Capoten ${ }^{x}$ is a registered trademark of E.R. Squibb \& Sons, Inc. Vasotec ${ }^{*}$ and Prinivit" are registered trademarks of Merck Sharp \& Dohme. Zestril" is a registered trademark of Stuart Pharmaceuticals.

\section{B A}

Taking the high cost of health care to heart 


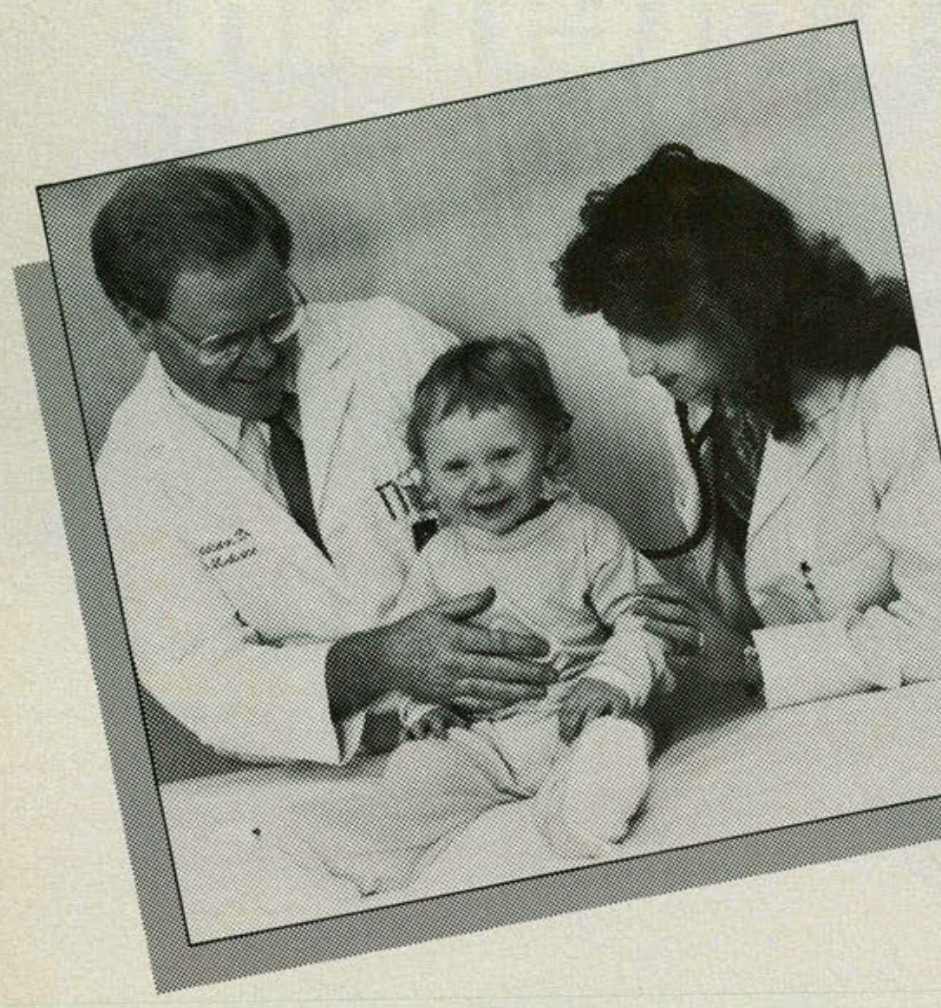

Your education

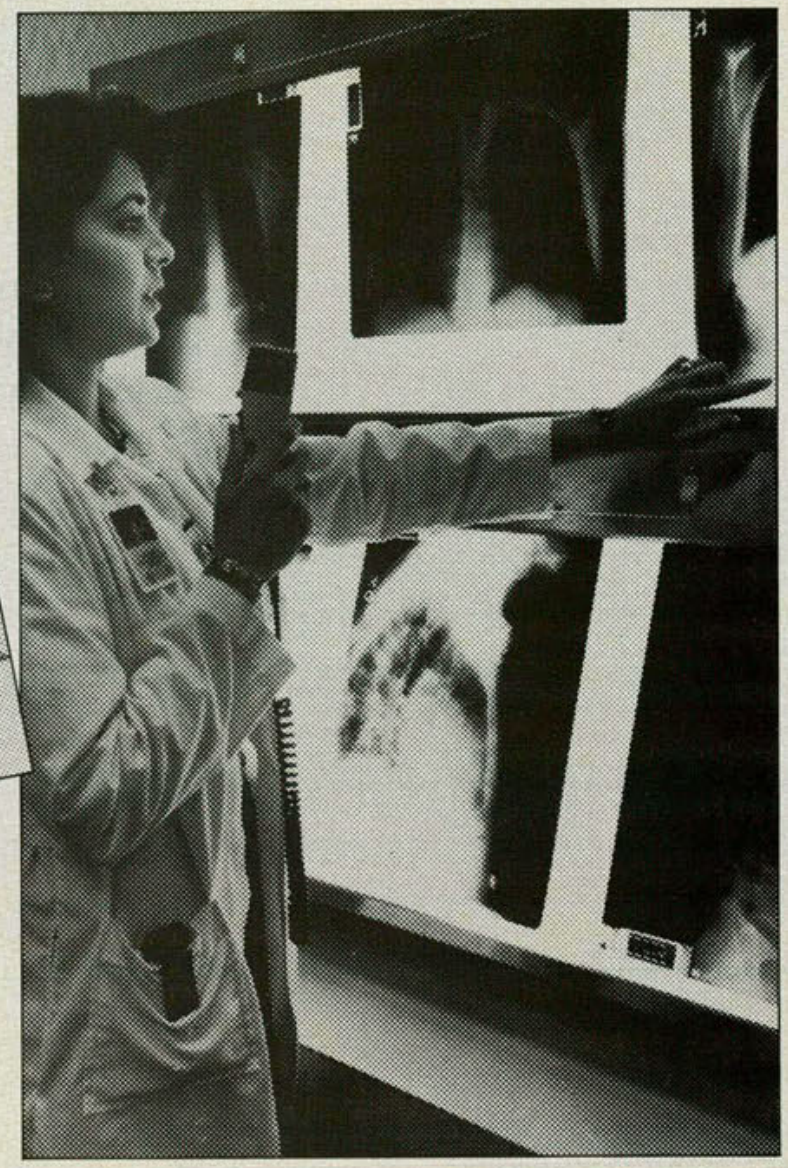

\section{is important to us!}

Left Pboto: Don Middleton, D.O., Resident, Tracy Middleton, D.O., Resident and "small" patient at FOH.

Right Pboto: Sucheta Kulkarni, D.O., Intern

"Flint Osteopathic Hospital has provided acute and general health care for the Flint community for 50 years. The hospital is affiliated with Michigan State University and offers a full range of medical, surgical, emergency, and intensive care services. All medical and surgical subspecialties are covered. At FOH the intern and resident receives training in both outpatient ambulatory care as well as inpatient practices.

FOH, with 359 beds, is the largest osteopathic hospital in Michigan offering intern and residency training programs for osteopathic physicians. The medical education program is designed to provide a structured curriculum and experience in diagnosis and treatment. Morning reports and guest physician lectures occur daily. Reading lists and objectives have been developed for each service. A monthly journal club is conducted by each clinical department. EKG conferences are scheduled twice monthly. The FOH Congdon Lecture Series brings both D.O. and M.D. physicians to the hospital each month. Prominent practitioners, representative of both medical communities, share expertise in research findings during these monthly, day-long seminar presentations.

Ambulatory clinics have been established and provide longitudinal continuity training for interns and residents. Both traditional and alternative track internships are available at FOH. The hospital is a charter member of the Consortium of Osteopathic Graduate Medical Education and Training (COGMET) in association with Michigan State University."
Residencies

- Anesthesia

- Family Practice

- Gastroenterology

- Internal Medicine

- Obstetrics/Gynecology

- Ophthalmology

- Orthopedics

- Otorhinolaryngology

- Pathology

- Pulmonary

- Radiology

- Surgery

- Urology

\section{Fellowships/Subspecialty Residencies}

- Medical Diseases of the Chest - Gastroenterology

One-year rotating internsbips Student externsbips

Christopber T. Meyer, D.O

Vice President of Medical Education

Dennis V. DeSimone, D.O. Director of Medical Education

(313) $762-4707$

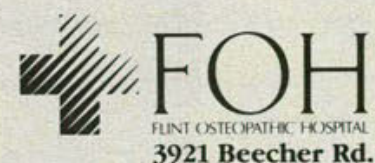

3921 Beecher Rd. Flint, MI 48532

\section{Where family matters}


